\title{
Considerações Metodológicas na Elaboração de Experimentos com Priming de Repetição
}

\author{
Juliana Burges Sbicigo ${ }^{1}$ \\ Programa de Pós-Graduação em Psicologia da Universidade Federal do Rio Grande do Sul, \\ Porto Alegre, RS, Brasil \\ Gerson Américo Janczura \\ Departamento de Processos Psicológicos Básicos da Universidade de Brasília, \\ Brasília, DF, Brasil \\ Jerusa Fumagalli de Salles \\ Departamento de Psicologia do Desenvolvimento e da Personalidade da Universidade \\ Federal do Rio Grande do Sul, Porto Alegre, RS, Brasil
}

\section{Resumo}

O paradigma experimental do priming permite lançar luz sobre diferentes aspectos dos processos de memória e possui diversas aplicações. Priming é um efeito de memória implícita, no qual há a recuperação não consciente de informação experienciada recentemente. No Brasil, o volume de pesquisas utilizando esta metodologia é pouco expressivo se comparado à literatura internacional. Buscando incentivar estudos nesse paradigma, o presente artigo tem como objetivo apresentar e discutir aspectos metodológicos relevantes à elaboração de experimentos com priming de repetição, com ênfase na seleção de materiais (software, estímulos normatizados para o Brasil e relevância de fatores psicolinguísticos) e na manipulação de variáveis (instruções e tempo de exposição dos estímulos, por exemplo). Concluiu-se que: a) PsycoPy é uma excelente opção de software de acesso livre, enquanto E-prime é uma excelente opção com exigência de licença; b) estímulos dos testes de priming precisam dispor de normas brasileiras, considerando aspectos psicolinguísticos e normas para as propriedades de estímulos pictórios; c) Stimulus Onset Asynchrony (SOA) curtos (menores que $300 \mathrm{~ms}$ ) associados à máscara são excelentes para investigar ativações automáticas e não conscientes de priming; e d) técnicas para reduzir a influência explícita devem ser utilizadas, como o Critério da Intencionalidade da Recuperação. São apresentadas recomendações para pesquisas futuras.

Palavras-chave: Priming de repetição, memória implícita, psicologia experimental, cognição.

\section{Methodological Considerations in the Elaboration with Repetition Priming Experiments}

\begin{abstract}
The priming experimental paradigm sheds light on different aspects of memory processes and has several applications. Priming is an implicit memory effect, in which there is no conscious retrieval information recently experienced. In Brazil, very few studies using this methodology comparing to the international literature is observed. Aiming to encourage studies to use this paradigm, the goal of this paper is to present and discuss methodological aspects relevant to the elaboration of repetition priming
\end{abstract}

Endereço para correspondência: Universidade Federal do Rio Grande do Sul, Instituto de Psicologia, Avenida Ipiranga, 2600, sala 114, Porto Alegre, RS, Brasil 90035-003. E-mail: julianasbicigo@gmail.com 
experiments emphasizing the selection of materials (software, standardized stimuli lists for Brazil and the relevance of psycholinguistic factors) and the manipulation of variables (instructions and exposure time of the stimuli, for example). The conclusion was that: a) PsycoPy is an excellent free software access option, while E-prime is an excellent option to license requirement; b) priming stimuli tests must have Brazilian norms, considering psycholinguistic aspects and norms for property pictorial stimuli; c) short Stimulus Onset Asynchrony - SOA (less than $300 \mathrm{~ms}$ ) associated with the mask are excellent to investigate automatic and not conscious priming activations; and d) techniques to reduce the explicit influence should be used as the Retrieval Intentionality Criterion. Finally, recommendations for future research are presented.

Keywords: Repetition priming, implicit memory, experimental psychology, cognition.

\section{Consideraciones Metodológicas en la Preparación de Experimentos con Priming de Repetición}

\section{Resumen}

El paradigma experimental de priming permite arrojar luz sobre diferentes aspectos de los procesos de memoria y tiene muchas aplicaciones. En Brasil, el volumen de investigación utilizando esta metodología es pequeño si se compara con la literatura internacional. Tratando de fomentar los estudios en este paradigma, este artículo tiene como objetivo presentar y discutir aspectos metodológicos relacionados con la preparación de los experimentos con cebado repetición, con énfasis en la selección de materiales (software, estímulos estandarizados a Brasil y la pertinencia de los factores psicolingüísticos) y la manipulación variables (instrucciones y tiempo de exposición del estímulo, por ejemplo). Se concluyó que: a) PsycoPy es una opción excelente software de libre acesso, mientras que E-Prime es una excelente opción para requisito de licencia; b) pruebas de priming estímulos deben tener normas brasileñas, $t$ eniendo en cuenta los aspectos psicolingüísticos y normas para los estímulos de propiedad pictórios; c) SOAs cortas (menos de $300 \mathrm{~ms}$ ) con la máscara asociada son excelentes para investigar las activaciones automáticas y no conscientes de priming; y d) técnicas para reducir la influencia explícita deben utilizarse como la Intencionalidad de Recuperación Criterio. Se presentan recomendaciones para la investigación futura.

Palabras clave: Priming de repetición, memória implícita, psicología experimental, cognición.

Priming consiste em um efeito de memória implícita, no qual ocorre a recuperação não consciente e não intencional de informação experienciada recentemente (Graf \& Schacter, 1985). Em estudos de priming de repetição, a memória implícita é demonstrada através de uma mudança na identificação, produção ou classificação de um item (estímulo) em decorrência de um encontro recente com esse item sem haver consciência da relação entre o momento da exposição inicial ao item e a recuperação posterior (Tulving \& Schacter, 1990). O encontro prévio facilita o processamento do item, o que significa que ele deverá ser recuperado de forma automática (Jacoby, 1991).
O paradigma experimental do priming foi introduzido na literatura em meados da década de 1970 (Cofer, 1967) para investigar a identificação e associação de palavras em testes que não exigiam consciência da aprendizagem. $\mathrm{Na}$ década seguinte, esse paradigma foi consolidado na pesquisa neuropsicológica quando indivíduos com memória explícita prejudicada ou com amnésia mostravam desempenho comparável ao de controles naqueles testes (Schacter, Dobbins, \& Schnyer, 2004). Essa dissociação entre memória implícita (priming) e explícita (recordação ou reconhecimento) também foi investigada em indivíduos saudáveis para verificar aspectos nos quais esses tipos de memória dissociavam- 
-se funcionalmente, conforme manipulações do tipo codificação, tempo de estudo e atenção dividida (Roediger \& McDermott, 1993). Nos últimos 30 anos, os avanços teórico-metodológicos no paradigma do priming e o advento das técnicas de imageamento cerebral têm permitido investigar em profundidade diferentes processos cognitivos envolvidos, por exemplo, na percepção visual e seus respectivos correlatos neurais (Segaert, Weber, Lange, Petersson, \& Hagoort, 2013).

O priming é tradicionalmente avaliado através de testes perceptuais ou conceituais (Roediger, 1990). Os primeiros dependem de caracte- rísticas superficiais (físicas [formas, cor, posição, etc.]) dos estímulos e são sensíveis a manipulações da informação perceptual (p.ex., visual vs. aditivo), como os testes de decisão lexical, completar fragmentos (palavras ou imagens), identificação perceptual (palavras ou imagens) e nomeação de figuras. Por outro lado, os testes conceituais exigem a análise e retenção do significado do estímulo, enfatizando atributos semânticos como no teste de produção de exemplar de categoria e questões sobre conhecimento geral. As Tabelas 1 e 2 caracterizam os testes clássicos de priming de repetição perceptual e conceitual, respectivamente.

\section{Tabela 1}

Testes Clássicos de Priming de Repetição Perceptual

Teste Perceptual Descrição

Decisão

Lexical

Identificação

Perceptual

Completar

Fragmentos de Palavras

Completar Fragmentos de Figuras

Completar Radical/ Tríade de Palavra
O priming nesse teste tem sido estudado nos paradigmas de identidade, de forma e semântico, porém somente o primeiro enquadra-se no priming de repetição. No priming de identidade, um prime é apresentado rapidamente (em milissegundos) seguido por uma máscara \#\#\# e o alvo. Prime e alvo são iguais, modificando-se o modo de escrita, como no caso de palavras [luva (prime); LUVA (alvo)] ou pseudopalavras [cafo (prime); CAFO (alvo)]. A tarefa é decidir se o alvo consiste em uma palavra ou pseudopalavra. $\mathrm{O}$ efeito de priming é observado quando há uma maior proporção de respostas corretas e menor tempo de reação para alvos precedidos por prime idêntico se comparados aos precedidos por prime diferente. Em geral, o prime não é visível, produzindo o efeito de priming subliminar (quando um estímulo, apresentado abaixo do limiar de consciência, facilita o processamento de um alvo).

Fase de estudo: é apresentada uma lista de estímulos (ex.: palavras, figuras de objetos comuns) que são seguidos individualmente por uma máscara \#\#\#. Fase de teste: os estímulos são expostos brevemente (entre 15-30 milissegundos, Greene, 1992) e eles devem ser identificados. Solicitase aos participantes para responder com a primeira palavra que lhes vier à mente quando encontram a palavra que é apresentada muito rapidamente. O efeito de priming ocorre quando uma maior proporção de palavras/figuras estudadas é lida/nomeada corretamente se comparado às palavras/figuras não estudadas e se há menor tempo de reação na resposta aos estímulos vistos na fase de estudo.

Fase de estudo: é apresentada uma lista de palavras (ex.: AVELÃ). Fase de teste: as palavras são expostas de forma fragmentada (A E A). O efeito de priming ocorre quando uma maior proporção de palavras vistas na fase estudo é completada acuradamente e/ou com menor tempo de reação se comparada às palavras não vistas.

Fase de estudo: é apresentada uma série de figuras. Fase de teste: as figuras são apresentadas com linhas e fragmentos faltando até que a figura seja identificada. Podem ser estabelecidos até oito níveis de fragmentação, com os níveis mais baixos contendo menos fragmentos. $\mathrm{O}$ efeito de priming é observado quando há maior probabilidade de identificar figuras vistas antes com menor nível de fragmentação (com menos informação da figura) do que figuras não vistas.

Fase de estudo: é apresentada uma lista de palavras (ex.: lente). Fase de teste: as palavras são expostas com as primeiras três letras (len__ ). O indivíduo deverá dizer a primeira palavra que vier à mente para completar o radical da palavra. $\mathrm{O}$ experimento é delineado de modo que dez ou mais palavras poderiam completar o radical. $\mathrm{O}$ efeito de priming é observado pela maior probabilidade de completar o radical com uma palavra que foi vista na fase de estudo em comparação ao acerto ao acaso de palavras novas (não vistas antes). 
Nomeação de Figura

Decisão de Objeto
Fase de estudo: um conjunto de figuras é nomeado. Fase de teste: as figuras são apresentadas rapidamente (abaixo do limiar da consciência) e elas devem ser nomeadas o mais rápido possível. $\mathrm{O}$ efeito de priming é observado quando há maior acurácia e/ou menor tempo de reação para figuras vistas no estudo se comparados às não vistas.

Fase de estudo: são apresentados uma série de objetos não familiares possíveis e impossíveis. Fase de teste: é necessário classificar cada objeto como possível e impossível. Metade dos estímulos estudados e não estudados representam objetos possíveis que podem existir como formas tridimensionais e metade representam objetos impossíveis que contém violações de borda ou superfície que impedem a formação de estruturas tridimensionais. O efeito de priming é observado quando há maior acurácia de classificação de objeto para objetos estudados do que não estudados.

Nota. Fonte: Roediger \& Geraci (2003); Roediger \& McDermott (1993).

Tabela 2

Testes Clássicos de Priming de Repetição Conceitual

Teste Conceitual Descrição

Verificação

da Categoria

Produção de Exemplar de Categoria

Conhecimento Geral

Associação de Palavras
Fase de estudo: é apresentada uma lista de exemplares de categorias (ex.: jasmim, pedra preciosa). Fase de teste: são apresentados pares de categoria-exemplar (pedra preciosa-jasmim) e deve ser respondido se o exemplar pertence àquela categoria (metade dos exemplares pertence à categoria e foram vistos na fase de estudo). $\mathrm{O}$ efeito de priming é observado quando há maior número de respostas corretas para pares em que o exemplar foi visto antes.

Fase de estudo: é idêntica à de Verificação de Categoria. Fase de teste: uma lista de nomes de categorias (metade nomes de categorias de exemplares vistos, ex.: flor, rubi) é apresentada. O indivíduo deve gerar o primeiro exemplar da categoria que vier à mente. $\mathrm{O}$ efeito de priming ocorre quando há maior probabilidade de gerar exemplares que foram vistos antes daquela categoria do que exemplares não vistos. Alternativamente, esse teste pode ser realizado apresentando cerca de seis exemplares de seis categorias aleatoriamente na fase de estudo e, na fase de tese, solicita-se um número de até oito exemplares por categoria. $\mathrm{O}$ efeito de priming ocorre quando, dentre esses oito exemplares, a maioria dos exemplares mencionados são aqueles vistos na fase de estudo.

Fase de estudo: é apresentada uma série de palavras. Fase de teste: são realizadas questões de conhecimento geral, das quais metade tem como resposta correta uma das palavras estudadas antes, enquanto a outra metade delas não tem qualquer relação com a lista estudada. $\mathrm{O}$ efeito de priming ocorre quando há maior probabilidade de responder com palavras estudadas versus não estudadas.

Essa é uma adaptação da tarefa de completar radical de palavras para verificar a aprendizagem de novas associações. Fase de estudo: são apresentados pares de palavras sem relação semântica (ex.: barba-dança, cama-vidro). Fase de teste: são mostrados três tipos de pares - intactos (palavras apresentadas com o par original, como barba-dan___, recombinados (cama-mot__) e pares com palavras novas. O efeito de priming para novas associações ocorre quando há maior facilitação na resposta aos pares intactos.

Nota. Fonte: Roediger \& Geraci (2003); Roediger \& McDermott (1993).

Os testes perceptuais ou conceituais devem relevar um efeito de priming, isto é, uma facilitação ao responder a itens vistos antes, que é revelada pela maior acurácia da resposta (acerto/erro) e/ou redução no tempo de reação - TR em milissegundos (Schacter, Chiu, \& Ochsner, 
1993). Em testes como identificação perceptual, completar radical de palavras e produção de exemplar da categoria, o efeito tem sido calculado pela média ou mediana de acertos em itens estudados (old) menos a média ou mediana de acertos ao acaso em itens não estudados (new), isto é, $\mathrm{M}_{\text {old }}-\mathrm{M}_{\text {new }}=\mathrm{M}_{\text {priming. }}$. Há variações no cálculo do efeito com TR, uma vez que pode ser analisada somente a média ou mediana para itens corretos (ex.: Lozito \& Mulligan, 2010) ou a diferença no TR para itens estudados (old) e não estudados (new), isto é, $\mathrm{M}_{\mathrm{TR} \text { new }}-\mathrm{M}_{\mathrm{TR} \text { old }}=$ $\mathrm{M}_{\text {TRpriming }}$ (ex.: Xiong, Franks, \& Logan, 2003). Em decisão lexical, a medida mais utilizada têm sido a média do TR para itens corretos (ex.: Kessler \& Moscovitch, 2013). No teste de priming para novas associações, é realizada a comparação entre o desempenho em pares intactos relativo a pares recombinados (Kinoshita, 1999).

O desenvolvimento de estudos experimentais com testes de priming de repetição exige cuidados metodológicos que vão desde a seleção dos estímulos que compõe os testes até o efeito da manipulação de variáveis. O presente artigo apresenta e discute esses aspectos metodológicos em duas seções: (a) materiais para a elaboração de experimentos de priming, com ênfase na seleção de softwares, estímulos pictórios e verbais, tarefas distratoras e secundárias; e (b) manipulação de variáveis e seus efeitos no priming, destacando desde os cuidados experimentais, como a calibração pré-experimental e as técnicas para reduzir a influência explícita, até a manipulação de variáveis, como instruções, tempo de exposição dos estímulos e forma dos itens entre estudo e teste.

\section{Materiais para Elaboração de Experimentos de Priming de Repetição}

\section{Softwares}

Os softwares mais utilizados para produzir experimentos de priming são o E-prime (Schneider, Eschman \& Zuccolotto, 2002), DMDX (Forster \& Forster, 2003), PsychoPy (Peirce, 2007), SuperLab (SuperLab, 2011) e MATLAB
(Math-Works, Natick, MA). DMDX e PsycoPy são softwares livres, enquanto os demais exigem licença. Esses programas possuem como funções básicas (Stangor, 2010):

1. Permitir ao experimentador indicar quais estímulos serão apresentados em ordem aleatória ou sequencial,

2. Designar as condições experimentais aleatoriamente para as diferentes condições experimentais e apresentar diferentes instruções e estímulos nas diferentes condições,

3. Apresentar uma variedade de estímulos como textos, gráficos, vídeo e áudio, que podem ser escolhidos aleatoriamente de listas, agrupadas em blocos, e colocadas em diferentes locais na tela;

4. Coletar respostas, incluindo formato fixo ou livre, em teclado, mouse, voz ou botão de entrada,

5. Medir precisamente o tempo de duração em que o estímulo é mostrado, assim como o tempo decorrido entre a apresentação de um estímulo e a resposta do participante e

6. Registrar todos os dados em um arquivo que pode ser importado em outros pacotes de programas. Um estudo recente (Garaizar, Vadillo, López-de-Ipiña, \& Matute, 2014) comparou E-prime, PsycoPy e DMDX e concluiu que: (a) os três possuem alta precisão e acurácia quanto ao tempo de exposição de estímulos visuais conforme configurado pelo experimentador, mas DMDX possui a vantagem de medir o tempo não apenas em milissegundos, mas também em ticks (ou pulso, medida mais próxima do tempo real), que reduz fontes de erro de mensuração; (b) E-prime e PsycoPy possuem uma interface bastante amigável, enquanto DMDX envolve maior dificuldade de programação com a sintaxe DMASTR; e (c) PsycoPy pode ser combinado com Graphical User Interface (GUI) para produzir experimentos mais sofisticados e pode ser rodado em outros sistemas além do Windows, como Linux e Mac OS X). O SuperLab é um programa de interface acessível, entretanto é considerado menos flexível que o E-prime para experimentos complexos em algumas funções, tal 
como realizar sobreposição de estímulos. MATLAB é um programa de alto desempenho, agregando diferentes linguagens de programação. A ele pode ser incorporada a Caixa de Ferramentas de Priming Mascarado (Masked Priming Toolbox), que fornece uma variedade de estímulos e máscaras, que podem ser parametrizados quanto ao tempo, tamanho, localização e orientação (Wilson, Tresilian, \& Schlaghecken, 2011). MATLAB é frequentemente usado em experimentos de priming com Ressonância Magnética Funcional (fMRI) e algumas ferramentas adicionais permitem análises avançadas de redes neurais (http://www.neurosolutions.com/products/nsmatlab/), embora outros programas, como o E-prime, também possam utilizar extensões para fMRI.

\section{Estímulos Pictórios}

Há disponíveis normas brasileiras dos estímulos desenvolvidos por Snodgrass e Corwin (1988), que consistem em imagens de animais e objetos comuns desenhados em preto no fundo branco. As normas atendem aos critérios de nomeação, familiaridade e complexidade visual para crianças e adultos (Pompéia \& Bueno, 1998; Pompéia, Miranda, \& Bueno, 2001, 2003). Em testes no paradigma de completar fragmentos, a fragmentação das imagens pode ser realizada com o programa ULTRAFRAG (Life Science Associates, Bayport, NY), que utiliza o método de Snodgrass, Smith, Feenan e Corwin (1987), removendo blocos de pixels (16 x 16) aleatórios da imagem. É possível fragmentar a figura em até oito níveis (nível 1 - mais fragmentado e nível 8 - menos fragmentado), porém utilizar até seis níveis já pode ser o suficiente, pois os níveis 7 e 8 já mostram a imagem praticamente completa.

\section{Estímulos Verbais}

Há listas de estímulos verbais normatizadas para o português brasileiro, nas quais são considerados diferentes aspectos psicolinguísticos (frequência, concretude, força associativa, entre outros) que, se não controlados, podem gerar efeitos indesejáveis de confusão nos resultados (Janczura, 2005). Estão disponíveis normas brasileiras de: frequência de palavras na língua para crianças (Pinheiro, 1996) e adultos (Kuhn, Abarca, \& Nunes, 2000; Sardinha, 2004); concretude de palavras (Janczura, Castilho, Rocha, van Erven, \& Huang, 2007); associação semântica de palavras para adultos (Buratto, Gomes, Prusokowski, \& Stein, 2013; Salles et al., 2008; Zortea \& Salles, 2012) e crianças (Salles, Holderbaum, \& Machado, 2009); associação semântica para 69 categorias naturais (Janczura, 1996); e contexto e associação semântica para 20 categorias naturais (Janczura, 2005). Normas de frequência, associação semântica, concretude e frequência de palavras são também encontradas em Stein e Gomes (2009). Estão ainda disponíveis 108 palavras de cinco letras normatizadas por Pompéia e Bueno (2006), que são direcionadas ao teste de completar radical de palavras.

Frequência. Quanto maior o uso da palavra na língua (frequência), maior o grau de automaticidade e velocidade do processamento (MacLeod \& Kampe, 1996). Por serem mais familiares, palavras de alta frequência exigem menos atenção e são processadas mais rápido que palavras de baixa frequência. Por serem menos familiares, palavras de baixa frequência devem aumentar mais em familiaridade com a repetição da exposição se comparadas às palavras de alta frequência. De fato, palavras de baixa frequência estiveram associadas a um maior efeito de priming perceptual em testes de decisão lexical, identificação de palavra, completar fragmentos de palavra e completar radical de palavra (ex.: Duchek \& Neely, 1989; Forster \& Davis, 1984; Kirsner, Milech, \& Standen, 1983; MacLeod \& Kampe, 1996). Testes conceituais como verificação da categoria mostraram-se, por outro lado, menos afetados pela frequência da palavra (Balota \& Chumbley, 1984). Normas de frequência de palavras são fornecidas em estudos brasileiros (Kuhn et al., 2000; Pinheiro, 1996; Sardinha, 2004).

Concretude. Palavras concretas possuem vantagem em relação às abstratas, com as pri- 
meiras sendo processadas mais rápida e acuradamente por serem registradas tanto no código lexical quanto sensorial, enquanto, as últimas, apenas no código lexical (Paivio, 1971). O efeito de concretude foi verificado em decisão lexical, com respostas mais rápidas para palavras concretas (Kounios \& Holcomb, 1994). Apesar do efeito de concretude ter sido pouco pesquisado em outros testes de priming, ele deve ser controlado devido a seu potencial de afetar o processamento dos itens e produzir resultados com viés. Normas de concretude para 909 palavras do português brasileiro estão disponíveis em Janczura et al. (2007).

Vizinhança Ortográfica. Se uma palavra alvo ("carta") puder ser modificada ao trocar uma letra ("carga") sem modificação da posição das demais letras, isso influenciará no processamento desse item. Em teste de identificação perceptual, por exemplo, foram cometidos mais erros em palavras com vizinhos ortográficos de maior frequência na língua e menor porcentagem de erros nas palavras com muitos vizinhos ortográficos (Justi \& Roazzi, 2012). Em decisão lexical, foi observado um efeito inibitório do número de vizinhos ortográficos e do número de vizinhos de maior frequência que a da palavra-alvo em crianças, enquanto esses aspectos exerceram efeito facilitador em adultos (Justi \& Pinheiro, 2008).

Força da Associação. Quanto maior for a força associativa (força pré-existente entre as palavras de um par) maior será a probabilidade de um alvo ser evocado a partir de uma pista em um teste de associação livre (Nelson, Dyrdal, \& Goodmon, 2005). Esse aspecto é importante, sobretudo na seleção de categorias naturais e seus respectivos exemplares na elaboração do teste de produção de exemplar da categoria. A força de associação dos exemplares às suas respectivas categorias pode ser fraca (até $10 \%$ ), média (10 a $24 \%$ ) ou forte (a partir de $25 \%$ ), conforme Coney (2002). Exemplares fortemente associados à categoria devem ser evitados, pois o efeito será melhor explicado pela força de associação pré-existente ao invés de ser pelo efeito de priming (Roediger \& Geraci, 2003).

\section{Tarefas Distratoras}

Essas tarefas são utilizadas no intervalo entre fases de estudo e teste. Elas têm como objetivo retirar os estímulos da fase de estudo da memória de trabalho. Podem ser de tipos variados, como testes de memória de trabalho (ex.: N-ba$c k$, span de dígitos, monitoramento de estímulos [palavras, dígitos, sons]) ou testes como geração de nomes de cidades a partir de radicais de palavras, dentre outras (ex.: Clarke \& Butler, 2008; Mulligan \& Hartman, 1996). O termo tarefa distratora também é cunhado para designar tarefas realizadas simultaneamente a testes de memória, mas, na literatura de priming, essa tarefa é frequentemente denominada de tarefa secundária.

\section{Tarefas Secundárias}

São realizadas concomitantes ao teste de memória, nesse caso, ao teste de priming. Tarefas secundárias têm sido tipicamente empregadas com o objetivo de sobrecarregar os recursos atencionais durante a realização do teste de priming e investigar se os processos nele envolvidos são automáticos. Essas tarefas também têm sido utilizadas para investigar o papel da memória de trabalho e seus subcomponentes no priming (ex.: Baqués, Saíz, \& Bowers, 2004). Tarefas secundárias comumente utilizadas são: monitoramento de sílabas ou dígitos, monitoramento de tons puros (em Hz), decisão de número par ou ímpar, decisão quanto a se uma palavra contém uma ou duas sílabas, adição de número ou carga de memória de curto prazo (ex.: sequências de estímulos são apresentadas antes de cada item do teste de priming e devem ser recordados após aparição do item). As tarefas secundárias podem ser divididas nas categorias: (a) fácil, se exigem baixa frequência de resposta, como as tarefas de monitoramento de sílaba ou dígito e não são utilizadas de forma sincrônica ao teste de memória e, (b) difícil, se elas exigem alta frequência de resposta, como as tarefas de adição de número e são sincrônicas ao teste de memória (Rohrer \& Pashler, 2003; Spataro, Cestari, \& Rossi-Arnaud, 2011). As tarefas secundárias difíceis têm afetado o priming em alguns estudos (ex.: Gabrieli et al., 1999; Mulligan, 2003). 


\section{Manipulação de Variáveis em Experimentos de Priming de Repetição}

Nessa seção, são apresentados desde cuidados metodológicos (calibração pré-experimental e técnicas para reduzir a influência explícita) à manipulação de variáveis. Dentre as variáveis passíveis de manipulação, destacam-se aqui as instruções, tempo de exposição dos estímulos e efeitos das mudanças no estímulo entre as fases de estudo e teste.

\section{Calibração Pré-Experimental}

A calibração permite verificar a sensibilidade do experimento de priming já no período pré-experimental. É interessante que a linha de base revele acertos entre $25 \%$ e $35 \%$. Em completar radical de palavra, por exemplo, a taxa de acertos de linha de base deve ser razoavelmente baixa para que os efeitos obtidos sejam devido ao priming. Se a taxa de acertos for muito alta, então é difícil obter priming devido ao efeito de teto. Se a taxa for muito baixa, significa que o teste está muito difícil e os efeitos de priming não poderão ser observados devido ao efeito chão. Em produção de exemplar da categoria, controlar a força de associação contribui para evitar tais efeitos. A calibração também permite verificar se a magnitude do efeito de priming é satisfatória. Em testes perceptuais, a magnitude tende a ser entre $20 \%$ e $40 \%$, enquanto em testes conceituais, entre $5 \%$ e $20 \%$ (Roediger \& Geraci, 2003)

\section{Instruções}

As instruções deverão minimizar a intencionalidade. O participante é instruído a realizar o teste com maior habilidade possível, mas nenhuma menção é feita sobre a necessidade recordar os estímulos em um momento posterior, o que atende ao Critério de Intencionalidade da Recuperação (Schacter, Bowers, \& Booker, 1989). Os participantes podem ser instruídos de que se trata de uma pesquisa de normatização de aspectos psicolinguísticos e que eles deverão realizar várias tarefas envolvendo palavras ou, se for um teste não verbal, a avaliação de imagens/figu- ras. Na fase de teste, a instrução pode ser de que o teste é parte de uma série de tarefas de filtro ou distratoras (parte de outro projeto) antes do teste mais importante (no caso o teste explícito, se for parte do estudo) ou simplesmente pode-se transmitir as orientações básicas necessárias para realizar o teste. A instrução fundamental na fase de teste é a de que se deve responder com "a primeira resposta que vier à mente" (Roediger \& McDermott, 1993).

\section{Tempo de Exposição dos Estímulos}

O tempo de exposição será manipulado ou controlado de acordo com os objetivos do estudo e o efeito esperado. Em geral, testes perceptuais como o de identificação perceptual requerem a realização de uma fase de calibração do tempo de exposição. Mulligan (2011), por exemplo, incluiu uma sessão de calibração, na qual a exposição dos estímulos foi testada em $16 \mathrm{~ms}$ e 32 ms para definir o tempo em que o participante alcançava entre $30 \%$ e $40 \%$ de acertos na identificação de palavras na fase de teste.

Exposição Subliminar: Tempos inferiores a $50 \mathrm{~ms}$ são também utilizados para verificar os efeitos do priming subliminar (Reingold \& Merikle, 1988), principalmente em testes de decisão lexical e identificação perceptual. Na decisão lexical, por exemplo, o tempo decorrido entre a apresentação do prime e a apresentação do alvo, chamado de Stimulus Onset Asynchrony (SOA), pode ser ajustado até o indivíduo relatar que não sabe se houve um estímulo prime, muito menos o que ele era. O SOA para estímulos linguísticos tem variado de $20 \mathrm{~ms}$ a $67 \mathrm{~ms}$ na literatura da área. Na faixa de 60 e $67 \mathrm{~ms}$, é possível verificar "algo que aparece antes do alvo", embora o estímulo não seja identificável (Forster, Mohan, \& Hector, 2003). Um SOA de $40 \mathrm{~ms}$ produziu um efeito de priming de identidade/repetição subliminar em decisão lexical (Busnello, Stein, $\&$ Salles, 2008). Dehaene et al. (2001) encontraram evidências de que, comparada à condição de primes visíveis, aquela com primes subliminares causou uma drástica redução da atividade neural a partir de $29 \mathrm{~ms}$, acelerando o processamento das respostas para primes e alvos idênticos (priming de identidade), em teste nomeação de palavras. 
Exposição dos Estímulos com Máscara (masked priming). Os estímulos, além de serem apresentados de forma sublimar, também podem ser pré-ativados com acréscimo de máscaras. Trata-se de um paradigma introduzido por Forster e Davis (1984), no qual cada tentativa começa com um ponto de fixação e é seguido por uma máscara (\#\#\#\#) e permanece na tela por $450 \mathrm{~ms}$ ou $500 \mathrm{~ms}$. Logo, o estímulo prime aparece por $50 \mathrm{~ms}$ e então é substituído pelo estímulo alvo (palavras, sons, imagens ou objetos). Um efetivo estímulo prime mascarado não deve ser relatado pelo observador porque ele não pode identificá-lo ou detectá-lo. Se a percepção ocorre sem consciência, então uma máscara efetiva não eliminará o efeito que o prime deve induzir (Kantowitz, Roediger, \& Elmes, 2009). É sugerido que, quanto maior o SOA, maior a detecção e a acurácia de identificação, pois haverá maior tempo para pensar sobre o prime e gerar expectativa quanto ao alvo. Pesquisas inicias (Altarriba \& Basnight-Brown, 2007) indicaram que um SOA de $300 \mathrm{~ms}$ foi capaz de inibir qualquer estratégia de expectativa, porém também foi demonstrado que estratégias operam em um SOA de $300 \mathrm{~ms}$. Posteriormente, foi verificado que estratégias não foram operantes em um SOA de $167 \mathrm{~ms}$. Logo, um SOA mais curto que $300 \mathrm{~ms}$ é necessário para evitar mecanismos de expectativa (Hutchison, Neely, \& Johnson, 2001). Cabe notar que a máscara também pode ser colocada após o estímulo (forward mask) e não somente antes (backward mask) dependendo do efeito desejado. A Caixa de Ferramentas para Priming Mascarado acrescida ao MATALB traz uma série de recursos para empregar máscara em experimentos (Wilson et al., 2011).

Mudanças na Forma do Estímulo. Mudar a forma física de um item entre as fases de estudo e teste reduz o efeito de priming, como quando a palavra é apresentada visualmente na fase de estudo e auditivamente na fase de teste (ex.: Jacoby \& Dallas, 1981). O efeito também é reduzido quando a mudança é na mesma modalidade perceptual (intramodal), como mostrado pelos efeitos de mudança na forma (caixa alta ou caixa baixa, torre - TORRE) das palavras (Graf \& Ryan, 1990; Roediger \& Blaxton, 1987) ou mudança na voz do falante quando as palavras foram ouvidas (ex.: Schacter \& Church, 1992). De forma similar, o efeito de priming de objetos foi maior quando os objetos foram mostrados na mesma forma física da fase de estudo do que quando foi mostrado um outro exemplar do mesmo objeto (Koutstaal et al., 2001), e quando os objetos foram idênticos no tamanho e ponto de vista se comparado a objetos idênticos, mas com tamanho e ponto de vista diferentes (Vuilleumier, Henson, Driver, \& Dolan, 2002). Essas evidências atestam a natureza altamente específica da memória implícita que sustenta o priming (Schacter et al., 2004; Tulving \& Schacter, 1990).

Técnicas para Reduzir a Influência Explícita. A intrusão de processos explícitos na recuperação implícita é um fenômeno plausível e deve ser considerado principalmente no desempenho de indivíduos saudáveis (sem lesão cerebral; MacLeod, 2008). A intrusão pode ocorrer em duas circunstâncias principais:

1. Os indivíduos percebem que sua memória está sendo testada e intencionalmente recuperam estímulos da fase de estudo enquanto realizam o teste de priming e

2. Os participantes seguem a instrução do teste e não se envolvem na recuperação intencional, mas, ainda assim, recordam os estímulos alvo, isto é, apresentam recordação involuntária (Stevens, Wig, \& Schacter, 2008). Na tentativa de minimizar a intrusão, alguns recursos metodológicos podem ser empregados (MacLeod, 2008; Roediger \& McDermott, 1993): (a) instruções de estudo que não remetam ao fato de que se trata de um teste de memória; (b) instruções de teste que enfatizem a necessidade de responder com a primeira resposta que vier à mente; (c) múltiplas tarefas distratoras (filtros) entre as fases de estudo e teste; (d) conjunto de itens relativamente grande (ex.: 50 itens); (e) proporção de itens estudados igual ou menor que $50 \%$ do total; (f) testes iniciando com itens de preenchimento que não fazem parte dos itens alvo para evitar efeitos de primazia e recência; (g) Critério da Intencionalidade da Recuperação; (h) 
Técnica de Reaprendizagem e Reserva; (i) Procedimento de Dissociação de Processos e (j) Procedimento de Pista Associativa, (k) índices de consciência pós teste. Os itens ( $\mathrm{g}$ ) a (k) serão detalhados a seguir.

Critério da intencionalidade da recuperação (Schacter et al., 1989). Essa técnica é amplamente utilizada na pesquisa atual com priming. Comparam-se os desempenhos em testes de memória implícita e explícita. Se uma variável é capaz de dissociar os dois testes (afetar um, mas não o outro teste), então o teste implícito não deve ser substancialmente influenciado por processos explícitos. Caso contrário, os dois testes apresentariam desempenho semelhante. Para que essa comparação seja efetiva, os testes implícito e explícito devem variar apenas na instrução enquanto todas as outras variáveis dos testes devem permanecer constantes.

Técnica de reaprendizagem e reserva (Ebbinghaus, 1964, aprimorada por Nelson, 1971). Envolve a aprendizagem intencional dos estímulos seguido de um intervalo de uma ou duas semanas. Após, é realizado um teste para avaliar a habilidade de lembrar os estímulos-alvo (divididos em itens esquecidos vs. lembrados), seguido de uma testagem na qual é contrastada a reaprendizagem de itens idênticos aos originais (mas que não foram recordados) com a aprendizagem de itens diferentes. A fundamentação lógica desse procedimento é que à medida que os itens estudados são esquecidos no teste pré-reaprendizagem e forem mais bem reaprendidos do que itens novos há evidência de memória residual. Os resíduos são vistos como não conscientes dado que o teste anterior falhou em mostrar recordação consciente dos itens alvo. Portanto, a recordação parece não ser a base para a reaprendizagem, indicando processamento implícito.

Procedimento de dissociação de processos - PDP (Jacoby, 1991, 1998). Tem o objetivo de medir contribuições separadas de processos implícitos e explícitos ao desempenho em um único teste de memória. Por exemplo, participantes estudam uma lista de estímulos e depois realizam dois tipos de teste com instruções de inclusão e exclusão. As pistas dos testes são equivalentes em ambos os testes. As instruções de "inclusão" são iguais às de memória explícita, enfatizando que o indivíduo precisa recordar itens vistos antes. Se eles não conseguem lembrar, são instruídos a adivinhar. Assim, o teste inclui a recordação intencional e se essa falha, então inclui recuperação automática por priming devido à familiaridade. No teste de "exclusão", há a restrição de não poder utilizar itens da lista de estudo para responder. A racional desse procedimento é que o desempenho na inclusão é dirigido por processos intencionais, enquanto o de exclusão, por processos automáticos não intencionais. Posteriormente, as duas condições são comparadas, uma em que ambos os processos operam juntos para aumentar o desempenho e uma em que os dois processos são colocados em oposição. Jacoby (1991) desenvolveu um modelo algébrico para comparar medidas de influências conscientes e não conscientes de memória. Uma revisão em português sobre o PDP, com suas aplicações e limitações, é encontrada em Ferreira, Reis, Orghian e Sôro (2013).

Procedimento de pista associativa (Nelson, McKinney, Gee, \& Janczura, 1998). Ao invés das instruções atenderem ao Critério de Intencionalidade e do objetivo ser a busca por dissociações, os participantes não são informados acerca da natureza dos testes. A variável manipulada é o número de palavras associadas (tamanho do conjunto) na memória de longo prazo (associações pré-existentes à realização da tarefa experimental). O tamanho do conjunto tipicamente afeta a memória implícita perceptual, mas não a memória explícita. Uma descrição detalhada desse procedimento foge ao escopo desse artigo, mas o leitor poderá consultar Nelson et al. (1998).

Índices de Consciência. Os índices ou questionários de consciência são compostos por perguntas que buscam identificar o grau em que o indivíduo observou a relação entre itens de estudo e teste (Mulligan, 2011). Essa técnica, portanto, visa a reduzir a influência explícita a posteriori. Exemplos de questões são: (a) Você notou alguma conexão entre os itens que você leu antes e o teste que acabou de realizar? Se assim for, o que você percebeu? (b) Se você notou que os itens correspondiam àqueles apresentados an- 
teriormente, você intencionalmente tentou usar os itens da primeira parte do experimento para responder aos testes? Se o indivíduo responder sim a essas questões, pode-se classificá-lo como consciente da relação entre os itens de estudo e teste. Esse tipo de questionário, contudo, não permite identificar acuradamente se uma dupla ou muitos itens foram afetados e/ou se foi no início ou no final da testagem. Ainda, há possibilidade dos indivíduos esquecerem o seu grau de consciência ou relatarem nenhuma consciência quando na verdade eles estavam conscientes (MacLeod, 2008). Apesar das limitações, os índices são úteis por permitirem a análise separada do desempenho de indivíduos baseado no seu tipo de consciência (conscientes $\mathrm{X}$ não conscientes) da relação entre itens de estudo e teste (Roediger \& Geraci, 2003).

\section{Discussão}

O presente artigo fornece orientações básicas para pesquisadores que pretendam delinear experimentos com priming. Em síntese, pode-se concluir que: (a) PsycoPy é uma excelente opção de software de acesso livre, enquanto E-prime é uma excelente opção com exigência de licença, ambos com interface amigável; (b) estímulos dos testes de priming precisam dispor de normas brasileiras, considerando aspectos psicolinguísticos (ex.: frequência, etc.) e normas para as propriedades de estímulos pictórios (ex.: complexidade visual); (c) SOAs curtos (menores que $300 \mathrm{~ms}$, Hutchison et al., 2001) associados à máscara são excelentes para investigar ativações automáticas e não conscientes de priming, enquanto SOAs maiores podem favorecer a participação de processos conscientes; e (d) técnicas para reduzir a influência explícita devem ser utilizadas em sua maioria, destacando-se o uso da instrução que não remeta ao fato de ser um teste de memória, a orientação de responder aos itens de teste com a primeira palavra que vier à mente e o uso do Critério da Intencionalidade da Recuperação.

Há uma série de aspectos a serem investigados no âmbito do priming como, por exemplo, as características do priming conceitual. As evi- dências em psicologia cognitiva e neurociências são controversas acerca de sua dissociação funcional em relação à memória explícita e quanto às bases neuroanatômicas (ex.: Wang, Lazzara, Ranganath, Knight, \& Yonelinas, 2010). Além da pesquisa básica com indivíduos saudáveis, o paradigma de priming pode ser utilizado em pesquisas neuropsicológicas e neuropsiquiátricas, uma vez que condições clínicas que afetam a memória explícita podem manter o aprendizado implícito preservado.

Cabe destacar que muitos dos elementos aqui elucidados podem servir de base à elaboração de experimentos com priming aplicados a construtos psicológicos, como as atitudes, estereótipos, personalidade e comportamento do consumidor, por exemplo. Contudo, independente da área, o pesquisador deve atentar às especificidades daquele campo de estudo a partir de excelentes exemplos da literatura, até mesmo porque as técnicas evoluem a partir de avanços proporcionados pelas pesquisas. Nesse contexto, o presente artigo poderá auxiliar na tomada de decisão acerca da seleção de materiais e manipulação de variáveis no paradigma de priming de repetição.

\section{Referências}

Altarriba, J., \& Basnight-Brown, D. M. (2007). Methodological considerations in performing semantic and translation priming experiments across languages. Behavior Research Methods, 39(1), 1-18. doi:10.1371/journal.pone.0123686

Balota, D. A., \& Chumbley, J. I. (1984). Are lexical decisions a good measure of lexical access? The role of word frequency in the neglected decision stage. Journal of Experimental Psychology: Human Perception \& Performance, 10, 340-357. doi:10.1037/0096-1523.10.3.340

Baqués, J., Sáiz, D., \& Bowers, J. (2004). Effects of working memory load on long-term word priming. Memory, 12(3), 301-313. doi:10.1080/09658210244000469

Buratto, L. G., Gomes, C. F. A., Prusokowski, T. S., \& Stein, L. M. (2013). Inter-item association norms for the Brazilian version of the emotional Deese/Roediger-McDermott paradigm. Psicologia: Reflexão \& Crítica, 26(2), 367-375. doi:10.1590/S0102-79722013000200017 
Busnello, R., Stein, L. M., \& Salles, J. F. (2008). Efeito de priming de identidade subliminar na decisão lexical com universitários brasileiros. Psico, 39(1), 41-47. Recuperado em http://revistaseletronicas.pucrs.br/ojs/index.php/revistapsico/article/view/1506/2794

Clarke, A. J. B., \& Butler, L. T. (2008). Dissociating word stem completion and cued recall as a function of divided attention at retrieval. Memory, 16, 763-772. doi:10.1080/09658210802261116

Cofer, C. N. (1967). Conditions for the use of verbal associations. Psychological Bulletin, 68, 1-12.

Coney, J. (2002). The effect of associative strength on priming in the cerebral hemispheres. Brain and Cognition, 50, 234-241. doi:10.1016/S02782626(02)00507-9

Dehaene, S., Naccache, L., Cohen, L., Le Bihan, D., Mangin, J. F., Poline, J. B., \& Rivière, D. (2001). Cerebral mechanisms of word masking and unconscious repetition priming. Nature, 4(7), 752-758. doi:10.1038/89551

Duchek, J. M., \& Neely, J. H. (1989). A dissociative word-frequency $\times$ levels-of-processing interaction in episodic recognition and lexical decision task. Memory and Cognition, 17, 148-162. doi:1 $0.1080 / 17588928.2012 .697454$

Ebbinghaus, H. (1964). Memory: A contribution to experimental psychology. New York: Dover.

Ferreira, M. B., Reis, J., Orghian, D., \& Sôro, J. (2013). Procedimento de dissociação de processos. Psicologia, 27(2), 145-166. Recuperado em http://www.scielo.mec.pt/pdf/psi/v27n2/ v27n2a06.pdf

Forster, K., \& Davis, C. (1984). Repetition priming and frequency attenuation in lexical access. Journal of Experimental Psychology: Learning, Memory, \& Cognition, 10, 680-698. doi:10.1037/0278-7393.10.4.680

Forster, K. I., \& Forster, J. C. (2003). DMDX: A windows display program with millisecond accuracy. Behavior Research Methods, Instruments, \& Computers, 35, 116-124. doi:10.3758/ BF03195503

Forster, K., Mohan, K., \& Hector, J. (2003). The mechanics of masked priming. In S. Kinoshita \& S. J. Lupker (Eds.), Masked Priming: The State of the Art (pp. 3-37). New York: Psychology Press.

Gabrieli, J., Vaidya, C., Stone, M., Francis, W., Thompson-Schill, S., Fleischman, D., ...Wilson,
R. (1999). Convergent behavioral and neuropsychological evidence for a distinction between identification and production forms of repetition priming. Journal of Experimental Psychology: General, 128(4), 479-498. Retrieved from http:// www.psych.upenn.edu/stslab/assets/pdf/gabrieli_etal.pdf

Garaizar, P., Vadillo, M. A., Lopez-de-Ipiña, D., \& Matute, H. (2014). Measuring software timing errors in the presentation of visual stimuli in cognitive neuroscience experiments. PlosOne, 9(1), 85-108. doi:10.1371/journal.pone.0085108

Graf, P., \& Ryan, L. (1990). Transfer-appropriate processing for implicit and explicit memory. Journal of Experimental Psychology: Learning, Memory, \& Cognition, 16, 978-992. doi:10.1037/0278-7393.16.6.978

Graf, P., \& Schacter, D. L. (1985). Implicit and explicit memory for new associations in normal and amnesic subjects. Journal of Experimental Psychology: Learning, Memory, and Cognition, 11, 501-518. doi:10.1037/0278-7393.11.3.501

Greene, R. L. (1992). Implicit memory tasks: Paradigms in the making. In R. L. Greene (Ed.), Human memory: Paradigms and paradoxes (pp. 172-192). Hillsdale, NJ: L. Erlbaum Associates.

Hutchison, K. A., Neely, J. H., \& Johnson, J. D. (2001). With great expectations, can two "wrongs" prime a "right"? Journal of Experimental Psychology: Learning, Memory, and Cognition, 27, 1451-1463. doi:10.1037//02787393.27.6.1451

Jacoby, L. L. (1991). A process dissociation framework - Separating automatic from intentional uses of memory. Journal of Memory and Language, 30(5), 513-541. doi:10.1016/0749$-596 \mathrm{X}(91) 90025-\mathrm{F}$

Jacoby, L. L. (1998). Invariance in automatic influences of memory: Toward a user's guide for the processdissociation procedure. Journal of Experimental Psychology: Learning, Memory, and Cognition, 24, 3-26

Jacoby, L. L., \& Dallas, M. (1981). On the relationship between autobiographical memory and perceptual learning. Journal of Experimental Psychology: General, 110, 306-340. doi:10.1037/00963445.110.3.306

Janczura, G. A. (1996). Normas associativas para 69 categorias semânticas. Psicologia: Teoria e Pesquisa, 12, 237-244. 
Janczura, G. A. (2005). Contexto e normas de associação para palavras: A redução do campo semântico. Paidéia (Ribeirão Preto), 15(32), $417-$ 425. doi:10.1590/S0103-863X2005000300011

Janczura, G. A., Castilho, G. M., Rocha, N. O., van Erven, T. J. C., \& Huang, T. P. (2007). Normas de concretude para 909 palavras da língua portuguesa. Psicologia: Teoria e Pesquisa, 23(2), 195204. doi:10.1590/S0102-37722007000200010

Justi, F. R. R., \& Pinheiro, A. M. V. (2008). O efeito de vizinhança ortográfica em crianças brasileiras: Estudo com a tarefa de decisão lexical. Interamerican Journal of Psychology, 42, 559-569. Recuperado em http://pepsic.bvsalud.org/pdf/ rip/v42n3/v42n3a16.pdf

Justi, F. R. R., \& Roazzi, A. (2012). Efeitos de vizinhança ortográfica no português brasileiro: Um estudo com a tarefa de identificação perceptual. Psicologia: Reflexão \& Crítica, 25(2), 301310. doi:10.1590/S0102-79722012000200012

Kantowitz, B. H., Roediger, H., \& Elmes, D. (2009). Experimental Psychology. Belmont, CA: Wadsworth.

Kessler, Y., \& Moscovitch, M. (2013).The involvement of strategic explicit processing in repetition priming in the lexical decision paradigm. Memory, 21, 366-376. doi:10.1080/0 9658211.2012 .728611

Kinoshita, S. (1999). Priming for novel associations: Evidence for an attentional component. Memory, 7, 385-404. doi:10.1080/741944918

Kirsner, K., Milech, D., \& Standen, E. (1983). Common and modality-specific processes in the mental lexicon. Memory \& Cognition, 11, 621-630. doi:10.3758/BF03198287

Kounios, J., \& Holcomb, P. J. (1994). Concreteness effects in semantic processing: ERP evidence supporting dual-encoding theory. Journal of Experimental Psychology: Learning, Memory, and Cognition, 20, 804-823. doi:10.1037/02787393.20.4.804

Koutstaal, W., Wagner, A. D., Rotte, M., Maril, A., Buckner, R. L., \& Schacter, D. L. (2001). Perceptual specificity in visual object priming: fMRI evidence for a laterality difference in fusiform cortex. Neuropsychologia, 39, 184 199. Retrieved from http://graphics.cs.cmu.edu/ courses/16-899A/2014_spring/thevisualworld/ KoutstaalSchacter2001Neuropsychologia.pdf
Kuhn, D. C. S., Abarca, E., \& Nunes, M. G. V. (2000). Corpus Nilc de português escrito no Brasil (NILC-TR-00-7). São Carlos, SP: Universidade de Federal de São Carlos.

Lozito, J. P., \& Mulligan, N. W. (2010). Exploring the role of attention during implicit memory retrieval. Journal of Memory and Language, 63(3), 387-399. doi:10.1037/0278-7393.22.1.132

MacLeod, C., (2008). Implicit memory tests: Techniques for reducing conscious intrusion". In J. Dunlosky \& R. A. Bjork, Handbook of metamemory and memory (pp. 245-263). New York: Psychology Press.

MacLeod, C. M., \& Kampe, K. E. (1996). Word frequency effects on recall, recognition, and word fragment completion tests. Journal of Experimental Psychology: Learning, Memory, and Cognition, 22, 132-142. doi:10.1037/02787393.22 .1 .132

Mulligan, N. W. (2003). Effects of cross-modal and intra-modal division of attention on perceptual priming. Journal of Experimental Psychology: Learning, Memory and Cognition, 29, 262-276. doi:10.1037/0278-7393.29.2.262

Mulligan, N. W. (2011). Conceptual implicit memory and environmental context. Consciousness and Cognition, 20(3), 737-744. doi:10.1016/j.concog.2010.11.008

Mulligan, N. W., \& Hartman, M. (1996). Divided attention and indirect memory tests. Memory and Cognition, 24, 453-465. doi:10.3758/ BF03200934

Nelson, T. O. (1971). Savings and forgetting from long-term memory. Journal of Verbal Learning and Verbal Behavior, 10(5), 568-576.

Nelson, D. L., Dyrdal, G. M., \& Goodmon, L. B. (2005). What is pre-existing strength? Predicting free association probabilities, similarity ratings, and cued recall probabilities. Psychonomic Bulletin \& Review, 12(4), 711-719. doi:10.1080/20 445911.2013.775120

Nelson, D. L., McKinney, V. M., Gee, N. R., \& Janczura, G. A. (1998). Interpreting the influence of implicitly activated memories on recall and recognition. Psychological Review, 105, 299324. doi:10.1037/0033-295X.105.2.299

Paivio, A. (1971). Imagery and verbal processes. New York: Holt, Rinehart, and Winston.

Peirce, J. W. (2007). PsychoPy-Psychophysics software in Python. Journal of Neuroscien- 
ce Methods, 162, 8-13. doi:10.1016/j.jneumeth.2006.11.017

Pinheiro, A. M. V. (1996). Contagem de freqüência de ocorrência de palavras expostas a crianças na faixa pré-escolar e séries iniciais do $1^{\circ}$ grau. São Paulo, SP: Associação Brasileira de Dislexia.

Pompéia, S., \& Bueno, O. F. A. (1998). Preliminary adaptation into Portuguese of a standardized picture set for use in research and neuropsychological assessment. Arquivos de Neuro-Psiquiatria, 56, 366-374. doi:10.1590/ S0004-282X2001000300004

Pompéia, S., \& Bueno, O. F. A. (2006). Um paradigma para diferenciar o uso de memória implícita e explícita. Psicologia: Reflexão e Crítica, 19(1), 83-90. doi:10.1590/S0102-79722006000100012

Pompéia, S., Miranda, M. C., Bueno, O. F. A. (2001). A set of 400 pictures standardized for Portuguese: Norms for name agreement, familiarity and visual complexity for children and adults. Arquivos de Neuro-Psiquiatria, 59(2B), 330-337. doi:10.1590/S0004282X2001000300004

Pompéia, S., Miranda, M. C., \& Bueno, O. F. A. (2003). Brazilian standardized norms for a set of pictures are comparable with those obtained internationally. Arquivos de Neuro-Psiquiatria, 61(4), 916-919. doi:10.1590/S0004-282 X2003000600005

Reingold, E. M., \& Merikle, P. M. (1988). Using direct and indirect measures to study perception without awareness. Perception and Psychophysics, 44, 563-575. doi:10.3758/BF03207490

Roediger, H. L. (1990). Implicit memory - Retention without remembering. American Psychologist, 45, 1043-1056. doi:10.1037/0278-7393.6.5.576

Roediger, H. L., \& Blaxton, T. A. (1987). Retrieval nodes produce dissociations in memory for surface information. In D. S. Gorfein \& R. R. Hoffman (Eds.), Memory and cognitive processes: The Ebbinghause Centennial Conference (pp. 349-379). Hillsdale, NJ: Erlbaum.

Roediger, H. L., \& Geraci, L. (2003). Conducting implicit memory research: A practical guide. In A. Wenzel \& D. Rubin (Eds.), A guide to implementing cognitive methods with clinical populations. Washington, DC: American Psychological Association Press.
Roediger, H. L., \& McDermott, K. B. (1993). Implicit memory in normal human subjects. In F. Boller \& J. Grafman (Eds.), Handbook of neuropsychology (pp. 63-131). Amsterdam, Holland: Elsevier.

Rohrer, D., \& Pashler, H. (2003). Concurrent task effects on memory retrieval: A cumulative latency analysis. Psychonomic Bulletin \& Review, 10, 96-103. doi:10.3758/BF03196472

Salles, J. F., Holderbaum, C. S., Becker, N., Rodrigues, J. C., Liedtke, F. V., Zibetti, M. R., \& Piccoli, L. F. (2008). Normas de associação semântica para 88 palavras do português brasileiro. Psico, 39(3), 362-370. Recuperado em http://revistaseletronicas.pucrs.br/ojs/index. $\mathrm{php} /$ revistapsico/article/view/3836/3380

Salles, J. F., Holderbaum, C. S., \& Machado, L. L. (2009). Normas de associação semântica de 50 palavras do português brasileiro para crianças: Tipo, força de associação e set size. Revista Interamericana de Psicologia, 43(1), 57-67. Recuperado em http://www.redalyc.org/articulo. oa? id=28411918007

Sardinha, T. B. (2004). Lingüística de corpos. Barueri, SP: Manole.

Segaert, K., Weber, K., Lange, F., Petersson, K., \& Hagoort, P. (2013) The suppression of repetition enhancement: A review of fMRI studies. Neuropsychologia, 51, 59-66. doi:10.1016/j.neuropsychologia.2012.11.006

Schacter, D. L., Bowers, J., \& Booker, J. (1989). Intention, awareness, and implicit memory: The retrieval intentionality criterion. In S. Lewandowsky, J. C. Dunn, \& E. Tulving (Eds.), Memory Systems (pp. 47-65). Cambridge, MA, Massachusetts Institute of Technology Press.

Schacter, D. L., Chiu, C.-Y. P., \& Ochsner, K. N. (1993). Implicit memory: A selective review. Annual Review of Neuroscience, 16, 159182. doi:10.1146/annurev.ne.16.030193.001111

Schacter, D. L., \& Church, B. A. (1992). Auditory priming: Implicit and explicit memory for words and voices. Journal of Experimental Psychology: Learning, Memory, and Cognition, 18(5), 915-930.

Schacter, D. L., Dobbins, I. G., \& Schnyer, D. M. (2004). Specificity of priming: A cognitive neuroscience perspective. Nature Reviews Neuroscience, 5(11), 853-862. doi:10.1038/nrn1534 
Schneider, W., Eschman, A., \& Zuccolotto, A. (2002). E-Prime reference guide. Pittsburgh, PE: Psychology Software Tools.

Snodgrass, J., \& Corwin, J. (1988). Perceptual identification thresholds for 150 fragmented pictures from the Snodgrass and Vanderwart picture set. Perceptual Motor Skills, 67, 3-36. doi:10.2466/ pms.1988.67.1.3

Snodgrass, J., Smith, B., Feenan, K., \& Corwin, J. (1987). Fragmenting pictures on the apple macintosh computer for experimental and clinical applications. Behavior Research Methods, Instruments, \& Computers, 19(2), 270-274. doi:10.3758/BF03203798

Spataro, P., Cestari, V., \& Rossi-Arnaud, C. (2011). The relationship between divided attention and implicit memory. Acta Psychologica, 136(3), 329-339. doi:10.1016/j.actpsy.2010.12.007

Stangor, C. (2010). Research methods for the behavioral sciences. Belmont, CA: Cengage Learning.

Stein, L. M., \& Gomes, C. F. de A. (2009). Normas brasileiras para listas de palavras associadas: Associação semântica, concretude, frequência e emocionalidade. Psicologia: Teoria e Pesquisa, 25(4), 537-546

Stevens, W. D., Wig, G. S, \& Schacter, D. L. (2008). Implicit memory and priming. In J. H. Byrne (Ed.), Concise learning and memory (pp. 6586). London: Academic Press.

SuperLab. (2011). Computer software. San Pedro, CA: Cedrus Corporation.
Tulving, E., \& Schacter, D. (1990). Priming and human memory systems. Science, 247, 301-306. doi:10.1126/science. 2296719

Vuilleumier, P., Henson, R. N., Driver, J., \& Dolan, R. J. (2002). Multiple levels of visual object constancy revealed by event-related fMRI of repetition priming. Nature Neuroscience, 5, 491499. doi:10.1038/nn839

Wang, W. C., Lazzara, M. M., Ranganath, C., Knight, R. T., \& Yonelinas, A. P. (2010). The medial temporal lobe supports conceptual implicit memory. Neuron, 68(5), 835-842. doi:10.1016/j. neuron.2010.11.009

Wilson, A. D., Tresilian, J., \& Schlaghecken, F. (2011). The masked priming toolbox: An open-source MATLAB toolbox for masked priming researchers. Behavior Research Methods, 43(1), 210-214. doi:10.3758/s13428-010-0034-z

Xiong, M. J., Franks, J. J., \& Logan, G. D. (2003). Repetition priming mediated by task similarity in semantic classification. Memory \& Cognition, 31, 1009-1020. doi:10.3758/BF03196122

Zortea, M., \& Salles, J. F. (2012). Estudo comparativo das associações semânticas de palavras entre adultos jovens e idosos. Psicologia: Teoria e Pesquisa, 28, 259-266. doi:10.1590/S010237722012000300001
Recebido: 23/06/2015

$1^{a}$ revisão: 06/12/2015

Aceite final: 25/12/2015 